\title{
Automatic Vehicle Accident Detection Based on GSM System
}

\author{
Kader H.A. Al-Shara \\ Department of Computer Engineering/ Al-Farabi University College \\ Kaderalshara@yahoo.com
}

\begin{abstract}
The evolution of technology has increased the rate of traffic accidents that occurs frequently causing loss of life and property. Therefore, the automatic traffic monitoring system gradually attracted the attention of researchers in improving traffic safety through the field of intelligent transport systems. In this paper, a cost-effective approach based on GSM system for automatic traffic incident detection is proposed. This paper provides an optimal solution to reduce the death rate by using the vibration sensor and GSM system; the implementation system is based on hardware (circuits) and software to build a graphical user interface (GUI) using LabView TM to process the data. Sensors are installed into the vehicles on each side of the vehicle. An SMS will be sent to the user after the accident. This system will assist in search and rescue vehicle involved in the accident.
\end{abstract}

Keywords: Vibration sensor, GSM, LabView, SMS.

\section{Introduction}

Nodaway, the vehicle accident was increasing day by day that causes the accident deaths among citizens of our country. It has also rise the traffic hazard and road accident because of high demand in automobiles. For example, Malaysia has placed at number 20 in its list of countries with the highest deaths due to route accidents by The World Health Ranking 2011. Studies at the Malaysia Institute for Road Safety (Miros) have shown that an average of 18 people were killed on the road every day, and that is expected to rise to 29 by 2020 . [1]. Individuals way of life is under high hazard in view by the lack of better emergency facilities accessible in our nation. However, in order to avoid this issue a smart electronics devise has been proposed. The designed system is used to detect the vehicle mischance with the assistance of vibration sensor. The warning system designed utilizing GSM module network to provide the car accident position and the message is sent transmitted which contains a port value with the assistance of GSM modem to the cell phone. The system consists of a portable GSM setup with LED display and an electronic circuit to detect the accident level. When the accident was detecting it, information message is transmitted to police, hospital and families with the location that occur and can provide fast medical treatment to the victim of vehicle accident. This alert message is sent to the rescue group in a brief span, which will assist in saving the valuable lives [2]. In order to implement this system, a synchronized system is needed for hardware development for the sensor and a system that is controlled by using software called LabVIEW. Thus, by receiving the SMS, the families and rescue will know that the accident happened at which location. The main aims of this project are to design and develop accident economic model. The main objectives are as follow: (i) To design and develop a schematic model for this system; (ii) To examine the system using Lab View software in order to detect the vehicle accident and transmit the location of the accident using GSM system.

\section{Related Work}

The Providing Accident Detection in Vehicular Networks during OBD-II Devices and Androidbased Smartphones is proposed by C.Prabha Jorge et al [2]. This proposed system is mainly an Android based application that displays the smart vehicle paradigm by sending SMS to be able to detect accidents during an (OBD-II) interface, to discover mishaps [2]. The application assesses this $G$ force of the individuals if there should be an occurrence of a frontal crash which is utilized together with airbag triggers to distinguish mischances. The approval response based sure detection is measured via sending specifics of the incident over each e-mail or SMS with position and then by an automobile telephone call towards the crisis administrations. Moreover, it is based on the previously published articles which have been listed in [3, 4]. These systems are used to detect an accident and regulate the traffic of cars in the traffic rules for easing to facilitate the orderly stream of traffic with work system available. Incident detection range and traffic violations are based on fuzzy logic, but the algorithm used to calculate the cycle time. Based on (DW) with (DWDC) is for other parameters. The paper proposes an Accident detection system as section of (TLC) system to solve the traffic problems at an intersection. Fuzzy Logic system collecting data related to zoning, cross ratio and paths and after that following up on. It requires as an 
important info based on the system which is the total number of vehicles in a specific place and the velocity of vehicles at certain paths, the remnant is finished up from that point. Moreover, this article focuses on the importance of the use of smart phones and how is the development for the application of the system of detection and analysis of accidents and irrigated violations and report car accidents at the moment of the accident information is gathered from amid the smartphone's accelerometer sensor and analyzed using Dynamic Time Warping (DTW) to decide the seriousness of the mishap, lessen false positives and to tell rescue team of call of the accident site. Besides that, accidents can be accessed through smartphone application over the Internet to provide accurate and reliable information concerning the accident. Dinesh Kumar et al proposed to find the vehicle accident location [5]. A system is proposed to increase traffic safety in terms of security and reliability. It used C8051F120 microcontroller and oscillation sensor. Here, the car holder will receive a SMS with information about the site of the vehicle at predetermined intervals during GSM. C. Prabha et al. In [6] Through GSM and GPS they are used in incident detection and automatic vehicle positioning. In paper; it is proposed to use an accelerometer sensors to apply car alarms in terms of speed, location and this dangerous driving is detected. In addition, it is useful in detecting the accident and the time of the accident through a vibration sensor and (MEMS) or accelerometer. NiravThakor et al. have proposed the detection of traffic accidents automatic based on ARM \& GPS or MEMS sensor [7].

\section{System Design}

\subsection{System Block daigram}

This system is separated into two parts which is vibration sensor part and GSM module part. Both parts are designed and developed by using LabView software. Both parts are connected by using NI DAQ and GUI (Graphical User Interface) programming which is LabView software. Fig 1 below displays structure of the block diagram of the accident detection system.

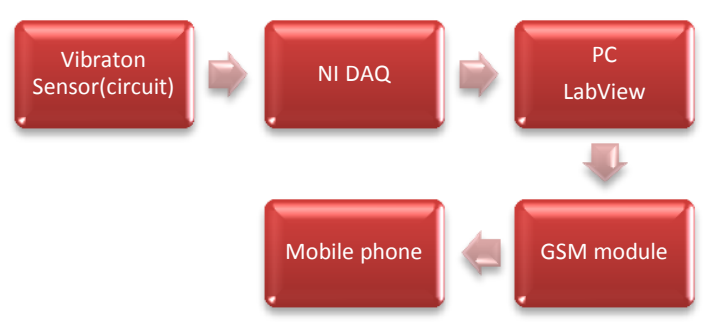

Figure (1): The block diagram of the accident detection system

Figure 2 shows the schematic diagram of the IC1 LM 358 is wired as an inverting Schmitt Trigger.
The R1 sets the threshold voltage of the circuit. R1 is used as a feedback resistor. The output of the piezo sensors is weak when it is not effective as well as do the output of the IC. In the case of activating the piezo sensor the high voltage results and the increase of Schmidt triggers. Therefore, the system buzzer will beep. The buzzer is kept for a short period of time although the vibration is removed because the increase in inverted input has an effect when the IC is triggered.

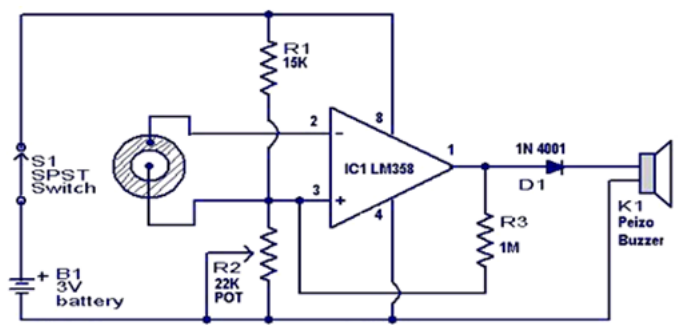

Figure (2): Schematic diagram for Accident Detection (Vibration Sensor)

\section{GSM Module and Labview}

This part is mainly based on GSM module via LabView Software. It is divided into two parts which is hardware part and software part. The GSM modem is 9001D RS232 GSM/GPRS modem that needs hyper terminal to set-up the connection to enable the GSM modem for sending message to hand-phone. Furthermore, LabView is used to build up a GUI to enable the GSM modem send message to mobile phone. However, AT command must set in the GUI block diagram and program it to send message to a mobile phone when the vibration sensor is triggered. Figure 3 shows how the GSM modem send message to mobile phone by using LabView.

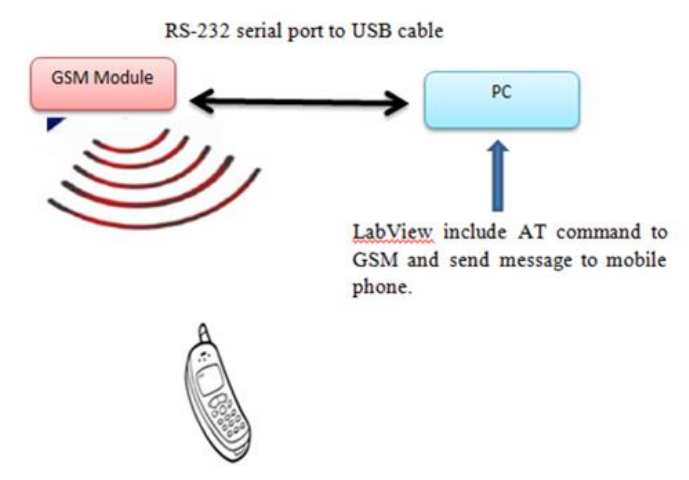

Figure (3) (a): GSM modem sending message to mobile phone by using LabView 


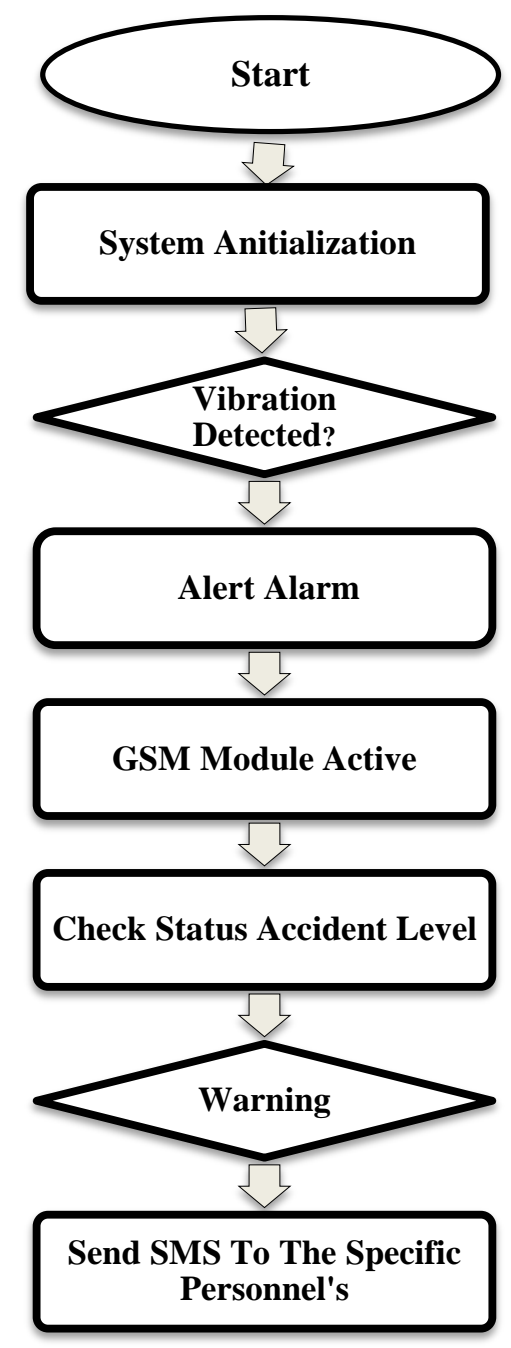

Figure ( 3)(b): Flow chart of system

\section{Hardware Design}

\subsection{A GSM Modem: MOD 900 ID RS232 GSM/ GPRS Modem}

The GSM modem is global System for Mobile Communication which is a wireless modem that works with GSM wireless network, and includes a range of sensors that used the radio waves digital interface to send and receive information.

This kind of GSM modem requires SIM card to be able to operate as digital identity to link with cellular phone network. GSM is used to set up cellular connection and for sending mobile voice and information services. GSM/GPRS can be transmitted by the computer serial port for wireless GSM communication including sending and receiving SMS. It can be connected to RS232 for remote management [11-13].

\subsection{NIDAQ 6008/6009}

Data acquisition on LabView is using the device called NI USB- 6008 or DAQ to capture any input signal or source any signal on the output port. The DAQ is important as the program of the block diagram is needed to collect the information from the outside world, also can send the output data of outside world with the data sent from the program. The data go through the DAQ into PC is usually analog signal data type where DAQ has the Analog-to-Digital converter and Digital-to-Analog converter on the device to convert the available data. NI USB-6008 and USB6009 is an easy and low-cost multifunction I/O product from National Instruments devices of the device [8]. The NI USB-6008 is DAQ and control device together with analog and digital which have input and output [9]. NI USB-6008 is a simple. The device contains the following features which are 8 analog inputs 12-bit, 2 analog outputs 12-bit, 12 digital I/O, USB connection, simply no extra powersupply is needed and NI-DAQmx driver software.

\subsection{Piezoelectric Sensor}

A piezoelectric is used to detect an accident. Very high piezoelectric transformer impedance output DC and relative voltage source. Piezoelectricity (also called the piezoelectric effect) could be looked of an electrical potential over the sides of a crystal when you subject it to mechanical pressure. Squeeze certain crystals and you can make electricity flow through them. They will "squeeze themselves" by vibrating backwards and forwards when you pass electricity through the same crystals. Moreover, the crystal turns into some sort of tiny battery using a negative charge on one shape and a positive charge on the other shape and the stream flows when the two shape connect together to make a circuit. From the reverse piezoelectric effect, a crystal becomes mechanically exhausting when a voltage is all over its opposite shape [10]. A transducer is a device that changes a small amount of energy from one kind into another. This is the features of piezoelectric [11]:

- Clear sound Ultra-thin and light

- No contacts: therefore, no noise and very reliable

- Reduced power usage for voltage type

The detailed mechanism of accident detection has been explained in [13].

\section{Experiment Results}

The project is designed via software and hardware component which shows the output of the 
component parameter control (such as vibration sensor), GSM module, NI DAQ 6008 and also the LabView programming. The proposed system will send warning message to user when the system is alerted. This will activate the vibration sensor to be triggered, LabView will send a command to GSM module to send a warning SMS to a particular person. AT command such as "at+cmgs="no number"" will write into LabView for sending message purpose. Figure 4 shows the front panel for the SMS control part.

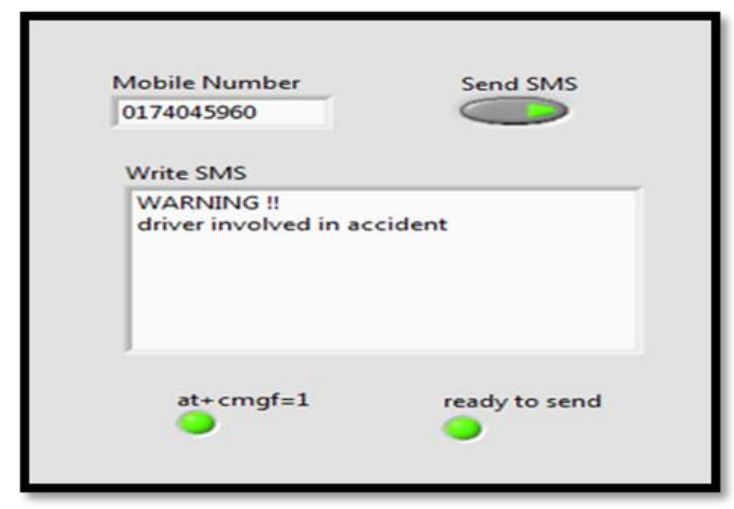

Figure (4): Front panel for SMS control

The message content which has been written in the SMS content box will be sent to the user mobile phone when the system is triggered. Figure 5-7 has shown the block diagram for the SMS control part including the overall hardware. The Standard RS-232 serial port cable is used to interface with PC USB port. All the components are connected to the breadboard.

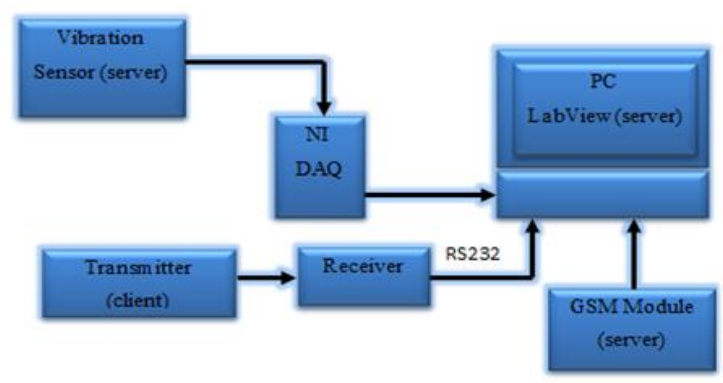

Figure (5): Block diagram of the SMS control part

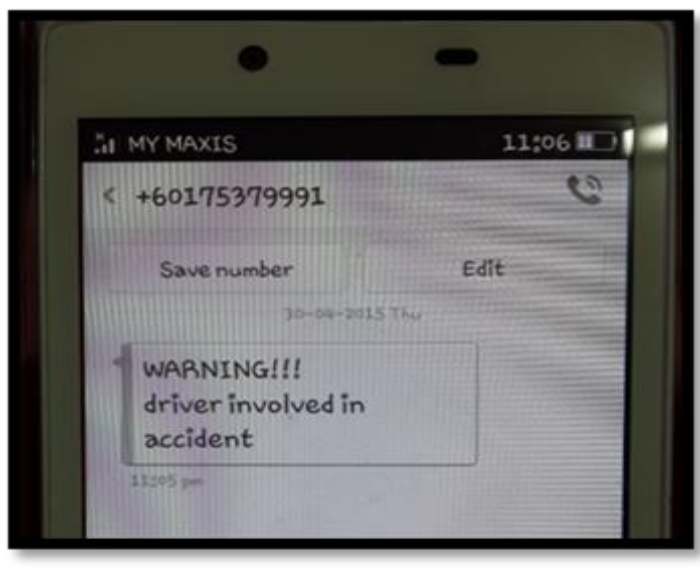

Figure (6): Sending SMS using HyperTerminal

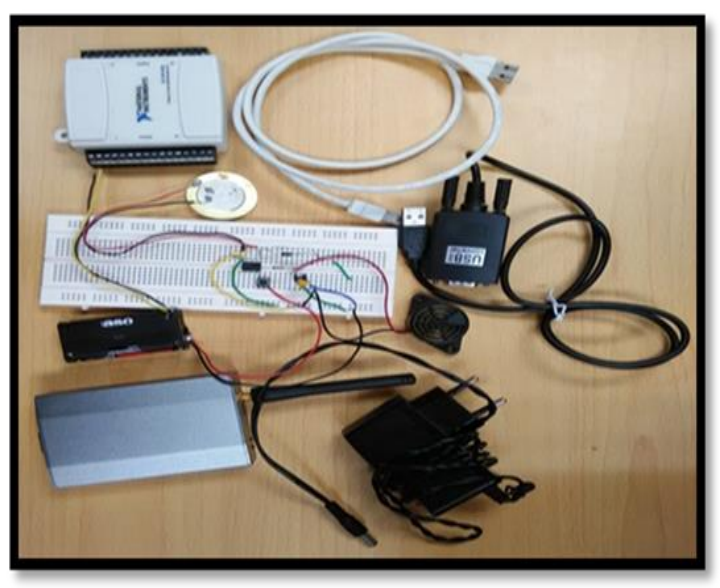

Figure (7): Hardware System

\section{Conclusion}

This paper presents the real-time, cost-effective hardware system of the automatic accident detection that is automatically alarmed, in such a way that the injured gets rescued in time. The automatic accident detection is suitable for different types of vehicles connected to it. This system is integrated between LabView software and hardware modeling. The system is capable of identifying and detecting accidents and sending SMS alert to specific persons. The SMS alert received by the user is on real-time incident occurrence and this feature allowed users to take action as quickly as possible. 


\section{References}

[1] Aina Nasa, Malaysia ranked 20th in road death, http://www.nst.com.my/node/11170, 23 Sep. 2014.

[2] National Instrument, labView environment basics, http://www.ni.com/gettingstarted/labView- basics/environment.htm, Vol. 24, issue.8, sep.2014M.

[3] Sky Microwave co. ltd. MOD 9001d RS232 GSM/GPS Modem user manual, National Instruments Corporation, user guide and specifications USB-6008/6009, 2007.

[4] P. Zhang, et al "Hardware design experiences in ZebraNet," in SenSys '12: Proceedings of the 2nd International Conference on Embedded Networked Sensor Systems. New York, NY, USA: ACM Press, 2012, pp. 227-238.

[5] Dinesh Kumar HSDK, Shreya Gupta... "Accident Detection and Reporting System Using GPS and GSM Module "JETIR ,Volume 2, Issue 5,2015.

[6] C. Prabha, R. Sunitha , R. Anitha "Automatic vehicle accident detection and messaging system using GSM and GPS modem" International Journal of advanced research in Electrical, Electronics and Instrumentation Engineering, Vol.3, Issue.12, 2014.

[7] NiravThakor, TanmayVyas "Automatic Vehicle Accident Detection System Based on ARM \&GPS"IJRTS, Vol-1, Issue - 1, Dec 2013.
[8] piezoelectricit,http://www.explainthatstuff.com /piezoelectricity.html, Vol. 5 October 2014. Chris Woodford.

[9] Abdulrahman Alkandari et al "Accident detection and action system using fuzzy logic theory", National Taiwan University of Science and Technology, Taipei, Taiwan, p. 1., 2013.

[10] N. Ramanathan, et al "Designing wireless sensor networks as a shared resource for sustainable development," in ICTD '06: Proceedings of the International Conference on Information and Communication Technologies and Development, May, pp. 256-265, 2006.

[11] J. Panchard, et al "Common-sense net: Improved water management for resource-poor farmers via sensor networks," in ICTD '06: Proceedings of the International Conference on Information and Communication Technologies and Development, May, pp. 22-33, 2009.

[12] M. Castillo-Effen, et al "Wireless sensor networks for flash-flood alerting," in Proceedings of the Fifth IEEE International Caracas Conference on Devices, Circuits and Systems. IEEE, pp: 142-146. Nov 2009.

[13] Hamid M. Ali "Car Accident Detection and Notification System Using Smartphone" IJCSMC, Vol. 4, Issue. 4, April 2015, pg.620 635. 